\title{
Sensitization to the mitochondrial pathway of apoptosis augments melanoma tumor cell responses to conventional chemotherapeutic regimens
}

\author{
RA Anvekarr ${ }^{1,2,3}$, JJ Asciolla ${ }^{1,2,3}$, E Lopez-Rivera ${ }^{1,4}$, KV Floros $^{1,3}$, S Izadmehr ${ }^{5}$, R Elkholi ${ }^{1,3}$, G Belbin $^{1,2,3}$, \\ AG Sikora ${ }^{*, 1,2,3,4}$ and JE Chipuk ${ }^{*, 1,2,3}$
}

Metastatic malignant melanoma is highly resistant to chemotherapy, and the average survival rate is under 1 year. The only FDAapproved conventional chemotherapy (i.e., dacarbazine) targets melanoma tumor cells by inducing a form of cell death referred to as apoptosis. However, dacarbazine exhibits a response rate of $\sim 5 \%$, and combination chemotherapies consisting of cisplatin, vinblastine, and dacarbazine often offer little clinical advantage over dacarbazine alone. Apoptosis is governed by the BCL-2 family of proteins, which is comprised of anti-apoptotic and pro-apoptotic members. To determine if the anti-apoptotic BCL-2 repertoire established the cell death threshold and chemoresistance in melanoma, a novel treatment strategy was designed to inhibit the anti-apoptotic BCL-2 members with ABT-737. Using various melanoma model systems, we determined the affects of ABT-737 on sensitivity to dacarbazine-based regimens. Strikingly, ABT-737 re-sensitized melanoma cell lines to common chemotherapeutics leading to marked BIM-mediated apoptosis. Cellular features of the ABT-737 combination treatments were loss of proliferation, mitochondrial fragmentation, nuclear condensation, phosphatidylserine exposure, and decreased clonogenic survival. We also observed significant anti-tumor activity in an in vivo melanoma model system. Our data indicate that ABT-737 may be a beneficial adjuvant therapy to improve melanoma response rates when conventional chemotherapy is the only option.

Cell Death and Disease (2012) 3, e420; doi:10.1038/cddis.2012.161; published online 15 November 2012

Subject Category: Experimental Medicine

Melanoma incidence is increasing, and while surgical removal of the primary tumor is often curative, the mean survival time following metastasis is rarely beyond 1 year due to chemotherapeutically resistant disease. ${ }^{1,2}$ Chemotherapy targets tumors by inducing a form of cell death called apoptosis, which is governed by the BCL-2 family of proteins and mitochondria. ${ }^{3}$ Apoptosis proceeds when the BCL-2 family compromises the outer mitochondrial membrane (OMM) allowing for pro-apoptotic factors (e.g., cytochrome c) within mitochondria to gain access to the cytoplasm, which leads to caspase activation and the apoptotic phenotype (reviewed in Chipuk and Green ${ }^{4}$ ).

The BCL-2 family is composed of two types of proteins: antiapoptotic and pro-apoptotic. ${ }^{5}$ Anti-apoptotic proteins (e.g., A1, BCL-2, BCL-w, BCL-xL, and MCL-1) preserve survival by binding and inhibiting the pro-apoptotic proteins. The pro-apoptotic members are divided into 'effectors' and the
'BH3-only proteins.' The effector proteins BAK and BAX homo-oligomerize into proteolipid pores at mitochondria to release cytochrome $\mathrm{c}$; however, this requires an activation step mediated by the 'direct activator' BH3-only proteins. ${ }^{6-8}$ BID and BIM are the major direct activators, and function via their $\mathrm{BH} 3$ domains to induce BAK/BAX activation and apoptosis. For the most part, the other BH3-only proteins (e.g., BAD, BIK, BMF, Noxa, and PUMA) only bind to antiapoptotic BCL-2 proteins and set the apoptotic threshold by releasing BID and BIM. ${ }^{9}$ Dynamic interactions between the anti- and pro-apoptotic proteins govern cell fate: promoting BID/BIM activity enhances apoptosis, while blocking them leads to survival and chemotherapeutic resistance. ${ }^{3}$

Conventional chemotherapy (e.g., dacarbazine) ideally targets melanoma tumor cells by inducing cell death to eliminate the tumor cells from the body. Yet, melanoma tumor cells have developed mechanisms to block BID/BIM from

\footnotetext{
${ }^{1}$ Mount Sinai School of Medicine Department of Oncological Sciences, One Gustave L. Levy Place, New York, NY, USA; ${ }^{2}$ Department of Dermatology, One Gustave L. Levy Place, New York, NY, USA; ${ }^{3}$ The Tisch Cancer Institute, One Gustave L. Levy Place, New York, NY, USA; ${ }^{4}$ Department of Otolaryngology, One Gustave L. Levy Place, New York, NY, USA and ${ }^{5}$ Department of Genetics and Genomic Sciences, One Gustave L. Levy Place, New York, NY, USA

${ }^{*}$ Corresponding author: Dr A Sikora, Mount Sinai School of Medicine Department of Oncological Sciences One Gustave L. Levy Place, Box 1189, New York, NY, 10029, USA. Tel: +212 659 9516; Fax: +212 369 5701; E-mail: andrew.sikora@mssm.edu

or Dr JE Chipuk, Mount Sinai School of Medicine Department of Oncological Sciences, One Gustave L. Levy Place, Box 1130, New York, NY 10029, USA. Tel: + 212659 5543; Fax: + 212849 2446; E-mail: jerry.chipuk@mssm.edu

Keywords: ABT-737; apoptosis; BCL-2 family; chemotherapy; melanoma

Abbreviations: OMM, outer mitochondrial membrane; BAX, BCL-2-associated x protein; BAK, BCL-2 antagonist killer 1; BCL-2, B cell CLL/Lymphoma-2; BCL-xL, BCL-2-related gene, long isoform; MCL-1, myeloid cell leukemia 1; BID, BCL-2 interacting domain death agonist; BIM, B-cell lymphoma 2 interacting mediator of cell death; STS, staurosporine; CVD, cisplatin/vinblastine/dacarbazine; Cis, cisplatin; Dac, dacarbazine; Vin, vinblastine; C8-BID, caspase-8 cleaved BID; siRNA, short interfering ribonucleic acid; SDS-PAGE, sodium dodecyl sulfate polyacrylamide gel electrophoresis; TUNEL, terminal deoxynucleotidyl transferase dUTP nick end labeling

Received 29.8.12; accepted 28.9.12; Edited by G Raschella'
} 
activating apoptosis following treatment. ${ }^{10} \mathrm{~A}$ mechanism of action for dacarbazine-based therapies is to kill tumor cells by DNA damage, and these treatments likely signal through the p53 pathway to induce apoptosis. ${ }^{11,12}$ Fortunately, p53 is rarely mutated in melanoma, so the pro-apoptotic arm of the p53 signaling pathway has potential to initiate. ${ }^{10}$ Yet, dacarbazine has been studied in multiple cellular models of melanoma, and the consistent observation is that dacarbazine promotes a relatively weak apoptotic response. ${ }^{13-16}$ In the current study, we aimed to increase melanoma responses to dacarbazine-based therapies by coordinated pharmacological inhibition of the anti-apoptotic $B C L-2$ repertoire with ABT-737. ${ }^{17}$ ABT-737 is a small molecule inhibitor to several anti-apoptotic BCL-2 family members, including BCL-2 and $\mathrm{BCL}-\mathrm{xL}$, and the drug is under investigation and evaluation for several other malignancies. ${ }^{17,18}$ Our aim was to identify if melanoma patients may benefit from ABT-737 as an adjuvant therapy.

\section{Results}

The murine B16 melanoma panel of cell lines (B16-F0, B16$\mathrm{F} 1$, and $\mathrm{B} 16-\mathrm{F} 10)$ is a highly tractable, reasonable approach to study chemotherapeutic responses as these cell lines are genetically matched, represent a variety of melanoma stages, and share several characteristics with human cell lines and primary melanomas. ${ }^{19,20}$ As such, B16-F0, B16-F1, and B16F10 represent primary, minimally metastatic, and highly metastatic cells, respectively, that robustly develop subcutaneous melanotic tumors and/or pulmonary melanoma metastases in vivo.

Patients with metastatic melanoma rarely respond to dacarbazine-based therapies, therefore we evaluated the response of the B16 lines to dacarbazine, and several other chemotherapeutics often used in combination with dacarbazine, such as cisplatin and vinblastine to validate our model system. ${ }^{21}$ Cells were treated with DMSO (vehicle), cisplatin (50 $\mu \mathrm{m})$, vinblastine (2 $\mathrm{nm}$ ), or dacarbazine (1 mM) for 24,48 , or $72 \mathrm{~h}$ before quantifying proliferation. As shown in Figure 1a, a time-dependent decrease in proliferation was evident for the dacarbazine treatment in B16-F0, but this sensitivity was reduced as metastatic potential increased. Furthermore, a similar sensitivity pattern was observed with cisplatin, and none of the cells exhibited altered proliferation with vinblastine treatment (Figure 1a).

We are interested in understanding the influence of the antiapoptotic BCL-2 repertoire on melanoma sensitivity to chemotherapy. To determine the potential for using BH3-only protein mimetics as an adjuvant therapy to promote chemotherapeutic responses in malignant melanoma, we evaluated ABT-737 in the B16 panel. Whole-cell lysates from the B16 panel were analyzed for anti-apoptotic BCL-2 family members by SDS-PAGE and western blot analyses. All the lines express multiple anti-apoptotic proteins, including BCL2, BCL-xL, and MCL-1 (Figure 1b); along with the proapoptotic effectors $B A K$ and $B A X$, suggesting that the panel is apoptosis-competent. ABT-737 inhibits BCL-2 and BCL-xL, so we hypothesized that ABT-737 should not kill the cells as one anti-apoptotic member remains functional. The B16 panel was treated with $\mathrm{ABT}-737$ and analyzed for changes to proliferation and survival. ABT-737 $(1 \mu \mathrm{m})$ caused no changes in proliferation after 24,48 , or $72 \mathrm{~h}$ (Figure 1a). Likewise, ABT-737 $(0.1,0.25,0.5,0.75,1 \mu \mathrm{m})$ did not induce apoptosis as measured by Annexin $\mathrm{V}$ staining and flow cytometry (Figure 1c; Supplementary Figure S1A and B). Staurosporine (STS; $100 \mathrm{~nm}$ ) treatment was used as a positive control in the apoptosis treatment and analyses. To determine if ABT-737 altered the expression profile of the anti-apoptotic BCL-2 proteins or pro-apoptotic BCL-2 effector proteins, which could potentially explain the lack of proliferation changes and apoptotic resistance, we chronically treated the B16 panel for 1 week with $1 \mu \mathrm{M}$ ABT-737 (or DMSO), and analyzed whole-cell lysates from the treated cells by SDS-PAGE and western blot. No changes to BAK, BAX, BCL-2, BCL-xL, or MCL-1 were observed after chronic ABT-737 treatment suggesting minimal toxicity (Figure 1d).

To determine the influence of anti-apoptotic BCL-2 family inhibition by ABT-737 on dacarbazine-induced apoptosis, we treated the B16 panel with this combination and quantified apoptosis $72 \mathrm{~h}$ later. Dacarbazine induced minimal, albeit dose-dependent apoptosis, with maximal responses between 10-25\% (Figure 2a). In combination with ABT-737, all the cell lines tripled their apoptotic responses to $30-75 \%$ (Figure $2 a$; Supplementary Figure S2A and B). To confirm the cell death pathway was apoptosis, we analyzed nuclear and mitochondrial morphologies after treatment using Höechst 33342 and MitoTracker Green staining, respectively. Dacarbazine and ABT-737 combination treatments lead to nuclear condensation and fragmentation (Figure $2 \mathrm{~b}$ ), along with mitochondrial fragmentation (Figure 2c). These phenotypes are associated with apoptosis, and their quantification (Figures $2 \mathrm{~b}$ and $\mathrm{c}$, right panels) parallels the Annexin $\mathrm{V}$ studies in Figure 2a. Importantly, nuclear condensation and mitochondrial fragmentation rarely occurred in dacarbazine or ABT-737 treatments alone (Figures $2 \mathrm{~b}$ and $\mathrm{c}$ ).

Dacarbazine is often used in combination with other drugs such as cisplatin and/or vinblastine. Therefore, we were interested in testing the effect of ABT-737 treatment on cisplatin and vinblastine-induced apoptosis, and on the triple combination often referred to as the cisplatin/vinblastine/ dacarbazine (CVD) regimen. The B16 panel was treated with various doses of vinblastine or cisplatin, in the presence or absence of ABT-737 for $48 \mathrm{~h}$ prior to Annexin $\mathrm{V}$ staining and flow cytometry. The combination of vinblastine and ABT-737 caused marked apoptosis in the B16-F0 line, but relatively little increases in B16-F1 and B16-F10 (Figure 3a; Supplementary Figure S3A and B). In contrast, the cisplatin and ABT-737 combination promoted marked apoptosis in all the cell lines (Figure 3b). ABT-737 co-treatment caused approximately double to triple the number of cell to undergo apoptosis.

All of our described cell death assays were performed by quantifying the percentage of cells undergoing apoptosis after $48 \mathrm{~h}$ of treatment. To determine if clonogenic survival was also decreased, we repeated the dacarbazine, vinblastine, and cisplatin treatments in the presence or absence of ABT-737 for $48 \mathrm{~h}$, changed the media, and allowed the cells to culture for an additional 10 days. As shown in Figure 4, clonogenic survival was unaffected by individual treatments of dacarbazine, vinblastine, cisplatin, or ABT-737; but the combination of 
a
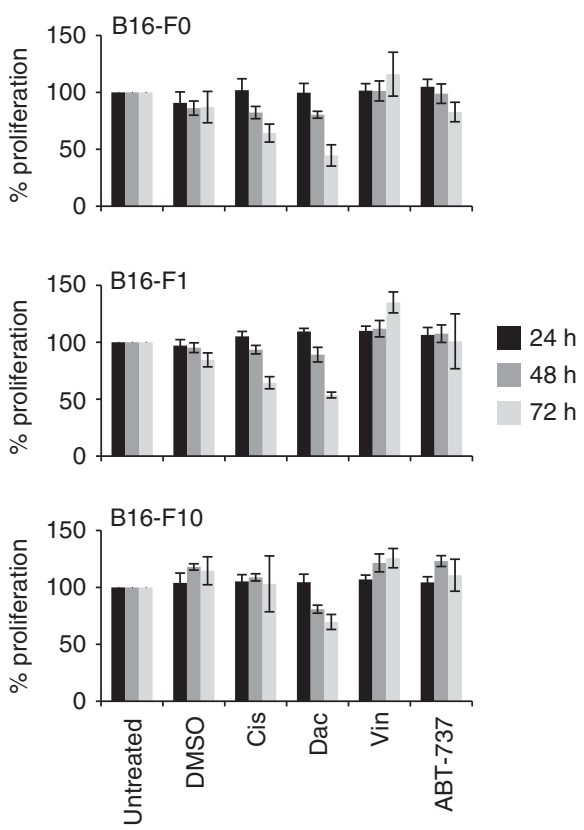

b
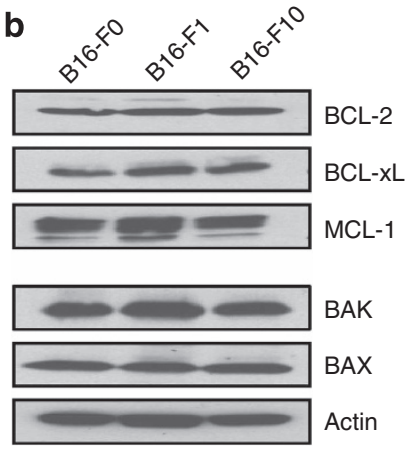

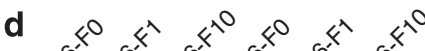
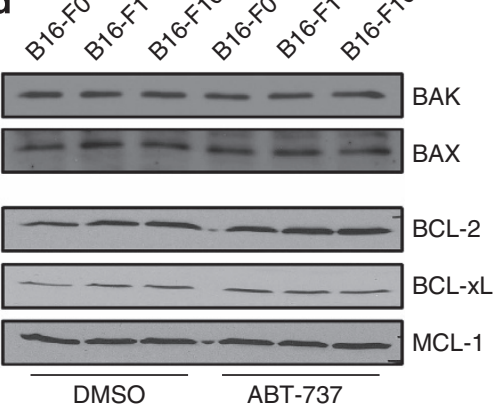

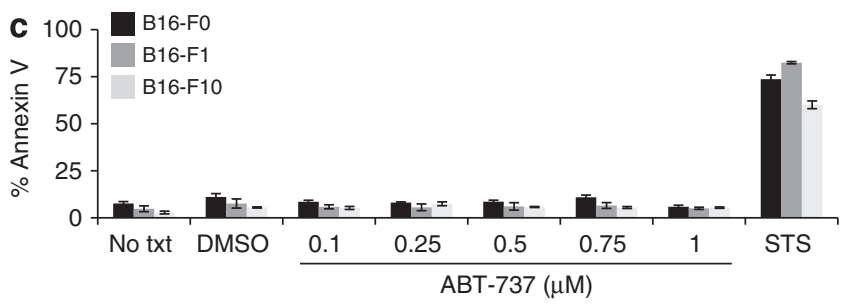

Figure 1 Cisplatin, vinblastine, dacarbazine, and ABT-737 as single treatments have minimal influences on cellular proliferation and survival in the murine B16 series. (a) Cells were treated with DMSO (vehicle), cisplatin $(50 \mu \mathrm{M})$, vinblastine $(2 \mathrm{nM})$, dacarbazine $(1 \mathrm{~mm})$, or ABT-737 $(1 \mu \mathrm{M})$ for 24,48 , and $72 \mathrm{~h}$ and analyzed for proliferation. (b) Whole-cell lysates where subjected to SDS-PAGE and western blot for anti-apoptotic (BCL-2, BCL-XL, MCL-1) and effector BCL-2 (BAK, BAX) proteins. Actin is the loading control. (c) Cells were treated with ABT-737 (doses indicated) or STS (100 nM, positive control) for $24 \mathrm{~h}$ before Annexin V staining and flow cytometry. (d) Cells were cultured for 7 days in the presence of ABT-737 $(1 \mu \mathrm{m})$ or DMSO before analysis by SDS-PAGE and western blot. All data are reported as \pm S.D. All experiments were performed in triplicate, at least

ABT-737 with either dacarbazine, vinblastine, or cisplatin caused a marked reduction in clonogenic survival (Figure $4 \mathrm{a}$ and b). Furthermore, the enhanced reduction in clonogenic survival exceeded the Annexin $\mathrm{V}$ positive populations after $48 \mathrm{~h}$ suggested some cells underwent cell death after the treatment was removed.

The CVD regimen is occasionally used instead of dacarbazine alone, as it has been suggested to confer slightly greater anti-tumor responses in clinical studies. We next wanted to evaluate if the CVD regimen response rates could be enhanced by ABT-737. To begin, we titrated cisplatin, vinblastine, and dacarbazine concentrations down to levels that caused no apoptotic response individually, but together there was an induction of apoptosis. The B16 panel was treated with low concentrations of cisplatin $(20 \mu \mathrm{M})$, vinblastine ( $1 \mathrm{~nm})$, and dacarbazine $(0.5 \mathrm{~mm})$ for $48 \mathrm{~h}$ prior to Annexin V staining and flow cytometry. Minimal apoptosis was observed following these individual treatments, which contrasted to the positive control in the assay, STS (Figure 5a). These low concentrations of cisplatin, vinblastine, and dacarbazine were combined into a 'CVD regimen' and added to the $B 16$ panel in the presence or absence of ABT-737, incubated for $48 \mathrm{~h}$, and the cells were stained with Annexin $V$ and quantified by flow cytometry. The CVD regimen induced modest apoptosis in the $\mathrm{B} 16$ panel, with the greatest response in the B16-F0 line, and the least response in the B16-F10 line, which parallels clinical data (Figure $5 \mathrm{~b}$ ). Similar to the data presented in Figures 2 and 3 , the combination of CVD and ABT-737 resulted in substantial cell death with an approximate three- to four-fold induction compared with CVD alone (Figure 5b). Importantly, the CVD + ABT-737 combination promoted the most significant enhancement of killing in the highly metastatic B16-F10 lines as compared with previous treatments (Figure $5 b$ ).

To determine which $\mathrm{BH} 3-$ only protein was induced by the CVD treatment explaining the synergy observed with ABT737, whole-cell lysates from DMSO or CVD-treated cells were analyzed by SDS-PAGE and western blot. After analyzing several BH3-only protein described to be involved in apoptosis (e.g., BAD, BID, BIM, PUMA, and Noxa), only BIM demonstrated CVD-mediated regulation, resulting in 

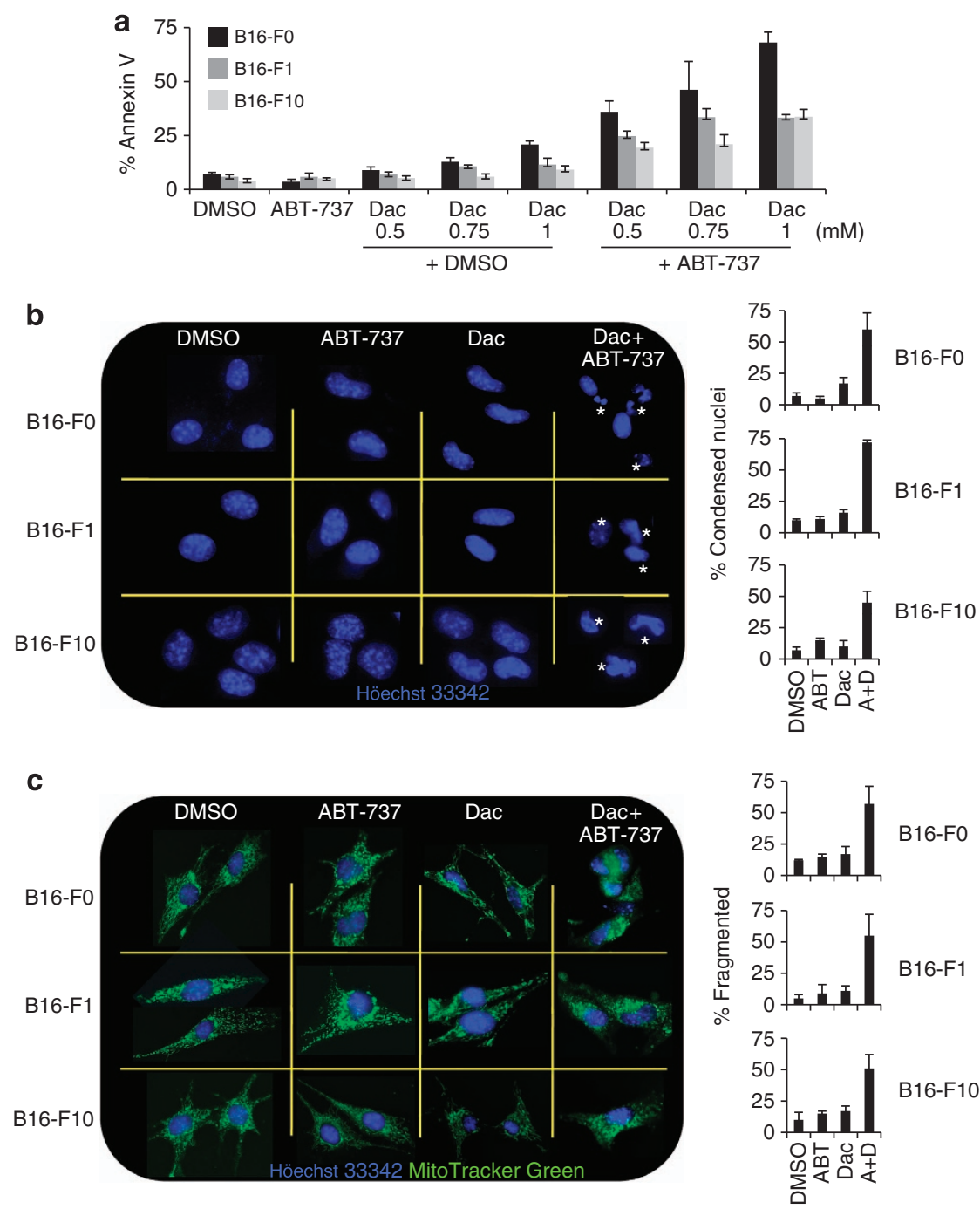

Figure 2 ABT-737 reveals a pro-apoptotic program induced by dacarbazine in the murine B16 series. (a) Cells were treated with dacarbazine $(0.5,0.75,1 \mathrm{~mm}) \pm \mathrm{ABT}-737$ $(1 \mu \mathrm{M})$ for $72 \mathrm{~h}$ before Annexin V staining and flow cytometry. (b, c) Cells were plated, treated with dacarbazine $(1 \mathrm{~mm}) \pm \mathrm{ABT}-737$ (1 $\mu \mathrm{m})$ for $24 \mathrm{~h}$ before loading with Hoechst 33342 (nuclear dye, blue) and MitoTracker (mitochondrial dye, green). The number of cell with condensed, apoptotic nuclei (b) and fragmented mitochondria (c) are presented. All data are reported as \pm S.D. All experiments were performed in triplicate, at least

substantially up-regulated expression (Figures $5 c$, and data not shown). Importantly, clonogenic survival of the CVD and ABT-737 treatments were markedly reduced compared with CVD and ABT-737 treatments alone, suggesting that proapoptotic signaling continues beyond short-term Annexin $\mathrm{V}$ studies (Figures $5 \mathrm{~d}$ and e; Supplementary Figure S4A-C).

Several mutations are associated with melanoma initiation and progression, such as $\mathrm{B}-\mathrm{RAF}^{\mathrm{V} 600 \mathrm{E}}$, which is present in $\sim 50 \%$ of patients. To determine if the CVD regimen in combination with ABT-737 would also enhance apoptosis in a human model of $B-R^{2} F^{\mathrm{V} 600 \mathrm{E}}$ positive melanoma, we examined this treatment in A375 cells. The A375 model expresses $B C L-2, B C L-x L$, and MCL-1 (Figure 6a), suggesting that ABT-737 will likely impact on apoptosis, as two of the three anti-apoptotic BCL-2 family members are responsive to ABT-737 inhibition. Furthermore, exposure of A375 cells to ABT-737 for $48 \mathrm{~h}$ did not result in detectable apoptosis (Supplementary Figure S5A), or changes to BCL-2, BCL-xL, or MCL-1 expression (Supplementary Figure S5B). As performed earlier, we titrated the cisplatin, vinblastine, and dacarbazine concentrations down to levels that caused no apoptotic response individually after $72 \mathrm{~h}$, which contrasted to the positive control in the assay, STS (Figure 6b; Supplementary Figure S6A-C). These low concentrations of cisplatin $(25 \mu \mathrm{M})$, vinblastine $(1 \mathrm{nM})$, and dacarbazine $(0.25 \mathrm{~mm})$ were combined into a CVD regimen, added to the A375 cells in the presence or absence of ABT-737, incubated for $72 \mathrm{~h}$, and the cells were stained with Annexin $V$ and quantified by flow cytometry. The CVD regimen induced timedependent apoptosis in the A375 cells, with a maximal response at $\sim 20 \%$ after $72 \mathrm{~h}$. In contrast, the addition of ABT-737 increased the percentage of apoptotic cells to $\sim 50 \%$ (Figure $6 \mathrm{c}$ ).

We analyzed the A375 mitochondrial network following CVD, ABT-737, and the combination treatment to further implicate the mitochondrial pathway of apoptosis. The A375 
cells were treated as indicated for $48 \mathrm{~h}$ in the presence of a pan-caspase inhibitor (zVAD-fmk, $100 \mu \mathrm{m}$ ) to allow for ease of imaging. After treatments, the cells were loaded with Höechst 33342 and MitoTracker Green. DMSO and ABT-737 treatments did not influence the mitochondrial network, but the CVD treatment yielded an irregular mitochondrial network
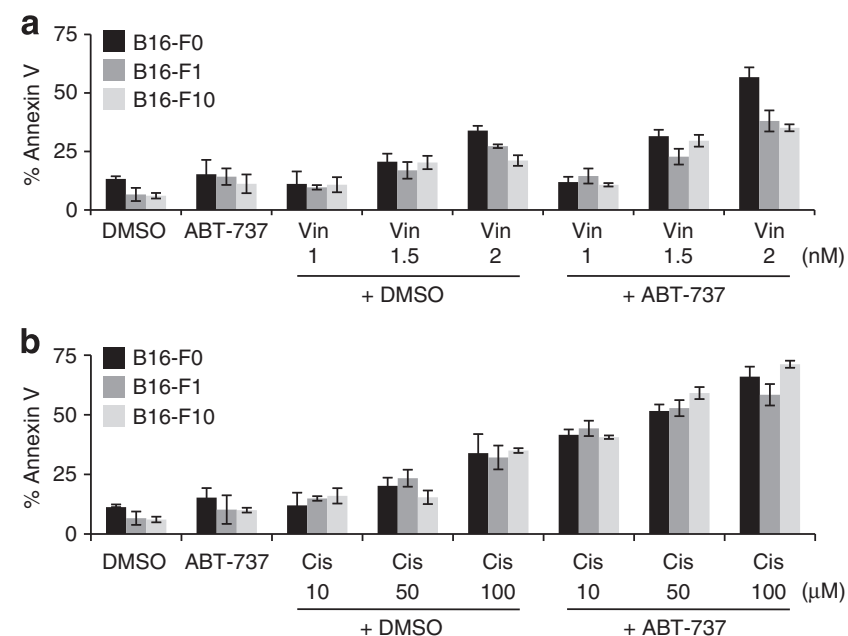

Figure 3 ABT-737 enhances the pro-apoptotic effects of various single agent chemotherapeutic treatments in the murine B16 series. (a, b) Cells were treated with vinblastine $(1,1.5,2 \mathrm{~nm})$ or cisplatin $(10,50,100 \mu \mathrm{M}) \pm \mathrm{ABT}-737(1 \mu \mathrm{m})$ for $48 \mathrm{~h}$ before Annexin $\mathrm{V}$ staining and flow cytometry. All data are reported as \pm S.D. All experiments were performed in triplicate, at least suggestive of stress (Figure 6d). In contrast, the combination treatment resulted in mitochondrial network fragmentation implicating BCL-2 family involvement in the cell death pathway (Figure 6d).

To determine the influence of the combination treatment on clonogenic survival of $A 375$, all of the drugs were added as either single agents (i.e., cisplatin, vinblastine, or dacarbazine), double agents (i.e., cisplatin + dacarbazine, dacarbazine + vinblastine, or cisplatin + vinblastine) or as the CVD regimen; and all of these combinations were evaluated in the absence and presence of ABT-737. As shown in Figure 6e, The CVD + ABT-737 combination treatment markedly decreased clonogenic survival of the A375 cell lines by nearly $85 \%$, suggesting long-term effects of the combination strategy on proliferation and survival (Figure 6e). Curiously, the single and double treatments did not regulate clonogenic survival, even in the presence of ABT737 , supporting the potential clinical utility of the CVD regimen over dacarbazine alone.

To provide mechanistic insight for the CVD regimen's ability to induce ABT-737-dependent apoptosis, we analyzed wholecell lysates from ABT-737 or CVD-treated A375 cells for changes to $\mathrm{BH} 3-$ only protein expression. Cells treated with CVD induced the expression of the potent pro-apoptotic protein, BIM (n.b., most likely all isoforms: BIM-EL, BIM-L, and BIM-S, compare long and short exposures for BIM triplet band, Figure 7a). The induction of BIM was absent in DMSO and ABT-737-treated cells (Figure 7a); as an aside, we did not analyze CVD + ABT-737-treated cells as they were

a
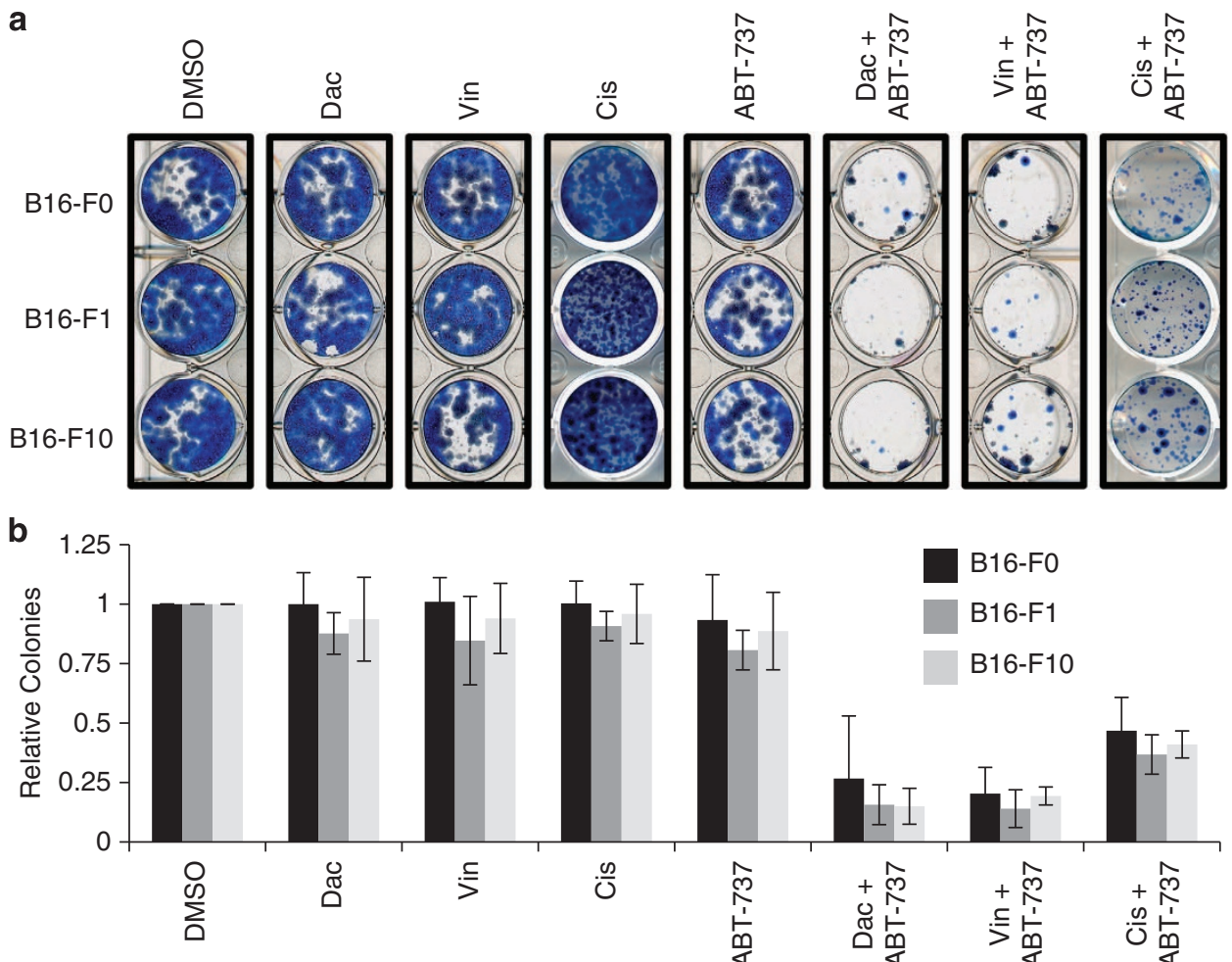

Figure 4 ABT-737 synergizes with dacarbazine, vinblastine, and cisplatin to decrease long-term clonogenic survival in the murine B16 series. (a, b) Cells were treated with dacarbazine $(1 \mathrm{mM})$, vinblastine $(2 \mathrm{~nm})$, or cisplatin $(100 \mu \mathrm{M}) \pm \mathrm{ABT}-737(1 \mu \mathrm{m})$ for $48 \mathrm{~h}$, the media was replaced, and the cells were cultured for an additional 10 days. The resulting colonies were then stained (a) and quantified (b). All data are reported as \pm S.D. All experiments were performed in triplicate, at least 


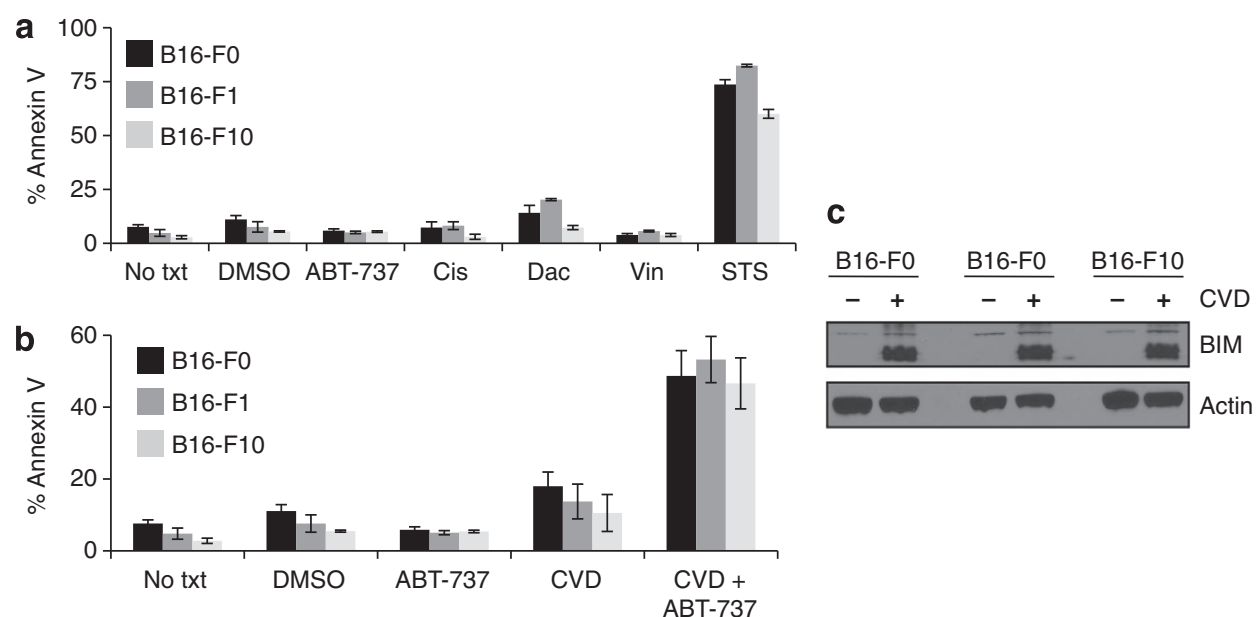

d
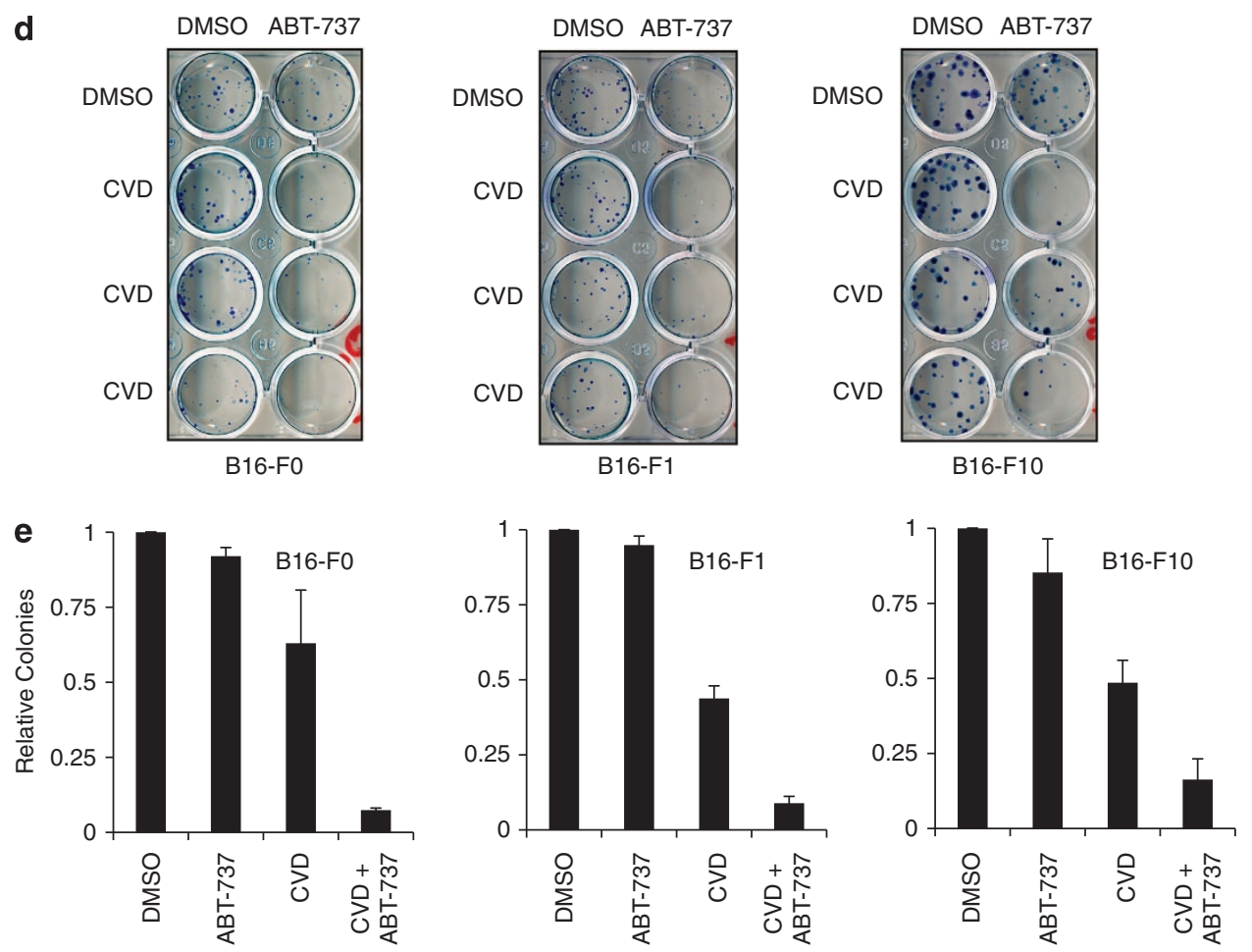

Figure 5 A chemotherapeutic regimen comprised of cisplatin, vinblastine, and dacarbazine induces BIM-mediated apoptosis when the anti-apoptotic BCL-2 repertoire is inhibited by ABT-737 in the murine B16 series. (a) Cells were treated with dacarbazine $(0.5 \mathrm{~mm})$, vinblastine $(1 \mathrm{~nm})$, or cisplatin $(20 \mu \mathrm{m}) \pm \mathrm{ABT}-737(1 \mu \mathrm{m})$ for $48 \mathrm{~h}$ before Annexin V staining and flow cytometry. (b) Cells were treated with the CVD regimen: dacarbazine ( $0.5 \mathrm{~mm})$, vinblastine (1 nM), and cisplatin $(20 \mu \mathrm{M}) \pm \mathrm{ABT}-737(1 \mu \mathrm{M})$ for $48 \mathrm{~h}$ before Annexin V staining and flow cytometry. (c) Cells were treated with DMSO or the CVD regimen for $48 \mathrm{~h}$, and resulting whole-cell lysates where subjected to SDS-PAGE and western blot for BIM and actin (loading control). (d, e) Cells were treated with the CVD regimen \pm ABT-737 $(1 \mu \mathrm{M})$ for $48 \mathrm{~h}$, the media was replaced, and the cells were cultured for an additional 10 days. The resulting colonies were then stained (d, triple data shown) and quantified (e). All data are reported as \pm S.D. All experiments were performed in triplicate, at least

undergoing massive apoptosis (Figure 6c). Furthermore, acute (data not shown) or chronic (Figure 7b) ABT-737 treatment up to $96 \mathrm{~h}$ did not alter the expression of BCL-2, $B C L-x L$, or MCL-1, suggesting that the specific induction of a pro-apoptotic BCL-2 protein is responsible for apoptosis, and not a decreased anti-apoptotic threshold mediated by ABT737 treatment.

The mitochondrial pathway of apoptosis is engaged at the $\mathrm{OMM}$ via the function of direct activator $\mathrm{BH} 3$-only proteins, such as BIM. CVD-induced BIM was hypothesized to accumulate on anti-apoptotic BCL-2 proteins localized to the OMM, and the addition of ABT-737 allowed for the release of BIM-S to mediated cytochrome c. To examine this activity, A375 cells were treated with DMSO or the CVD regimen for $24 \mathrm{~h}$, and the cells were permeabilized with digitonin to gain biochemical access to mitochondria. The permeabilized cells were treated with various doses of ABT-737 for 45 minutes at $37^{\circ} \mathrm{C}$, pelleted, and the supernatant was evaluated for the presence of cytochrome $\mathrm{c}$. Mitochondria from DMSO-treated cells did not release cytochrome $c$ after ABT-737 addition, but 
a

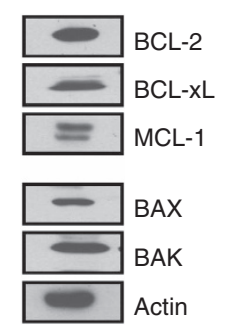

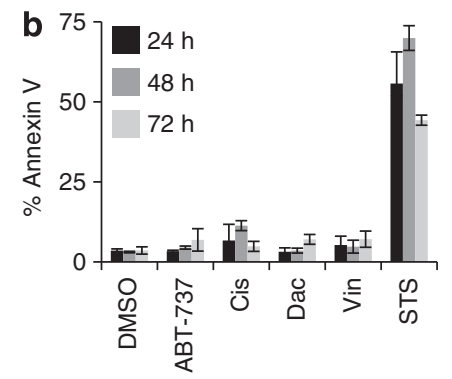
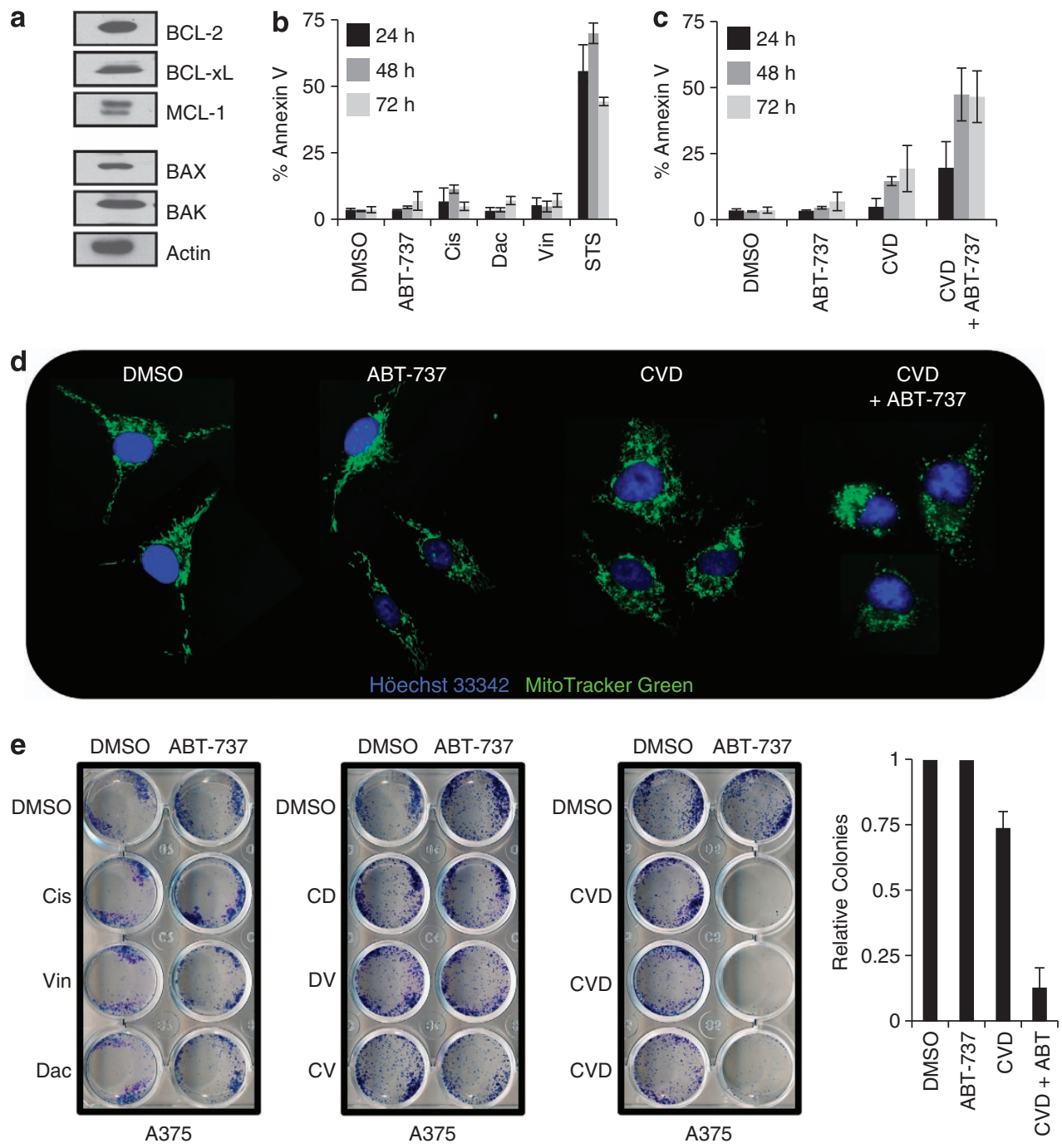

Figure 6 A chemotherapeutic regimen comprised of cisplatin, vinblastine, and dacarbazine induces apoptosis when the anti-apoptotic BCL-2 repertoire is inhibited by ABT-737 in the human A375 malignant melanoma model. (a) Whole-cell lysates where subjected to SDS-PAGE and western blot for anti-apoptotic (BCL-2, BCL-xL, MCL-1) and effector BCL-2 (BAK, BAX) proteins. Actin is the loading control. (b) Cells were treated with dacarbazine $(0.25 \mathrm{~mm})$, vinblastine $(1 \mathrm{nM})$, or cisplatin $(25 \mu \mathrm{M}) \pm \mathrm{ABT}-737$ $(1 \mu \mathrm{M})$ for up to $72 \mathrm{~h}$ before Annexin V staining and flow cytometry. (c) Cells were treated with the CVD regimen: dacarbazine $(0.25 \mathrm{~mm})$, vinblastine (1 nM), and cisplatin $(25 \mu \mathrm{M}) \pm \mathrm{ABT}-737(1 \mu \mathrm{M})$ for up to $72 \mathrm{~h}$ before Annexin V staining and flow cytometry. (d) Cells were plated, treated with the CVD regimen $\pm \mathrm{ABT}-737(1 \mu \mathrm{m})$ for $48 \mathrm{~h}$ before loading with Hoechst 33342 (nuclear dye, blue) and MitoTracker (mitochondrial dye, green). (e) Cells were treated with one (cisplatin $25 \mu \mathrm{m}$, vinblastine $1 \mathrm{~nm}$, or dacarbazine $0.25 \mathrm{~mm}$ ) or two drugs (Cis \& Dac, Dac \& Vin, or Cis \& Vin), or the CVD regimen, all \pm ABT-737 $(1 \mu \mathrm{m})$ for $48 \mathrm{~h}$, the media was replaced, and the cells were cultured for an additional 10 days. The resulting colonies were then stained (left panels) and CVD treatments \pm ABT-737 were quantified (right panel). Triple data are shown for CVD \pm ABT737. All data are reported as \pm S.D. All experiments were performed in triplicate, at least

CVD-treated cells released nearly $100 \%$ of their cytochrome c after ABT-737 addition (Figure 7c). As positive control for both cell treatments, recombinant caspase-8 cleaved BID (C8BID) was added and resulted in $100 \%$ cytochrome c release, suggesting that mitochondria from DMSO-treated cells maintain the capacity to undergo $\mathrm{BH} 3$-only protein-induced cytochrome $\mathrm{c}$ release (Figure $7 \mathrm{c}$ ). To ensure that BIM expression was sufficient to cooperate with ABT-737 to promote cytochrome $c$ release, untreated $A 375$ cells were permeabilized and the $\mathrm{BH} 3$ domain peptide derived from BIM was added in the presence or absence of ABT-737 and cytochrome $c$ release was measured. At low concentrations, the BIM BH3 domain peptide induced minimal cytochrome $\mathrm{C}$ release; but when $\mathrm{ABT}-737$ was added in addition to the BIM
$\mathrm{BH} 3$ domain peptide, cytochrome c release was robust and complete (Figure 7d), indicating that the presence of BIM is necessary and sufficient to cooperate with ABT-737 to promote apoptosis.

The above data suggest that BIM is required for CVDinduced apoptosis in the presence of ABT-737. To directly examine this requirement, the expression of bim was silenced using RNAi in A375 cells, followed by treatment with $C V D \pm A B T-737$ for $24 \mathrm{~h}$. The treated cells were then harvested for both western blot and apoptosis determination. As shown in Figure 7e and f, A375 'control siRNA' cells treated with the CVD regimen induced $\mathrm{BIM}$ expression, and demonstrated the expected sensitivity to the CVD + ABT-737 treatment. This is in contrast to the 'bim siRNA' cells, which demonstrated neither 

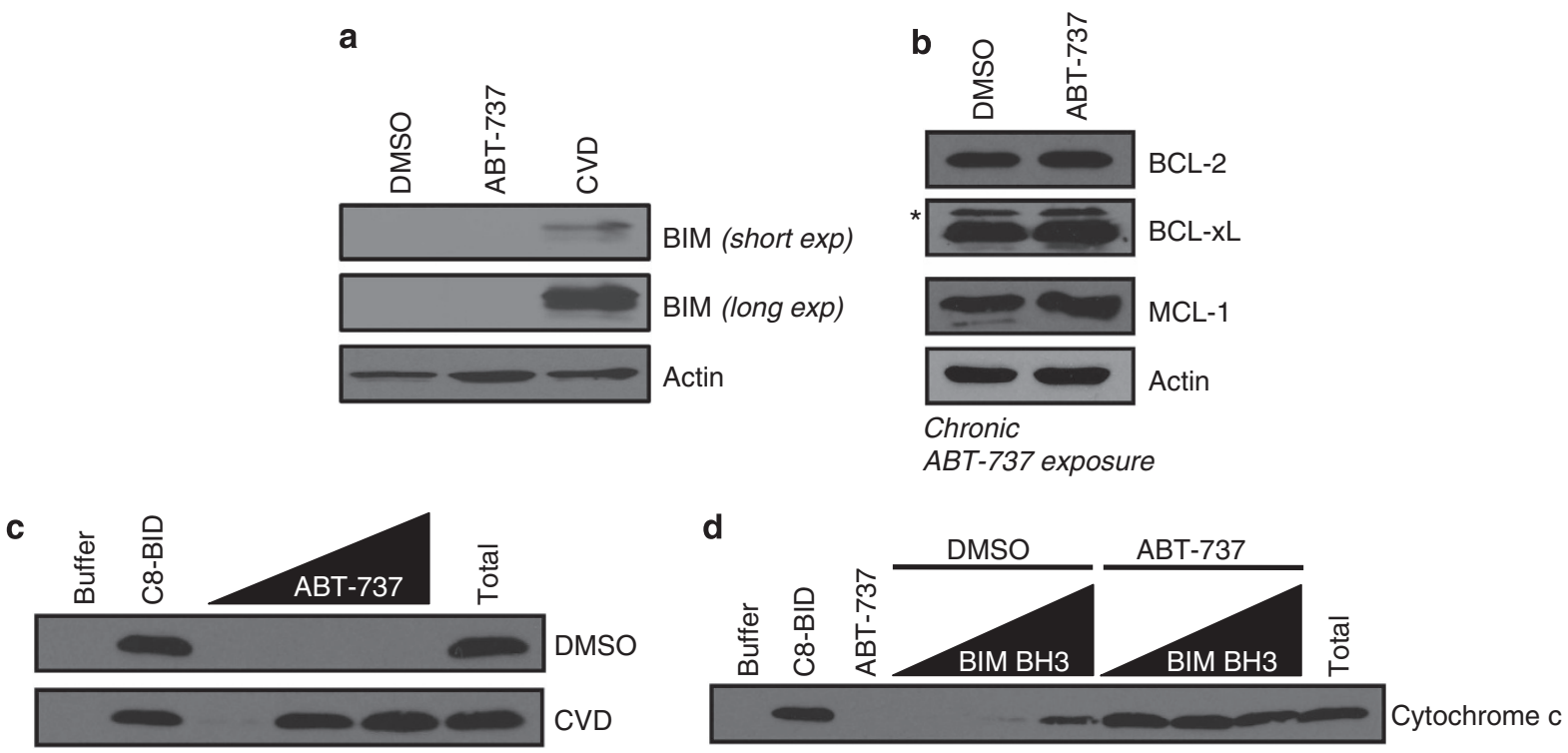

Cytochrome c
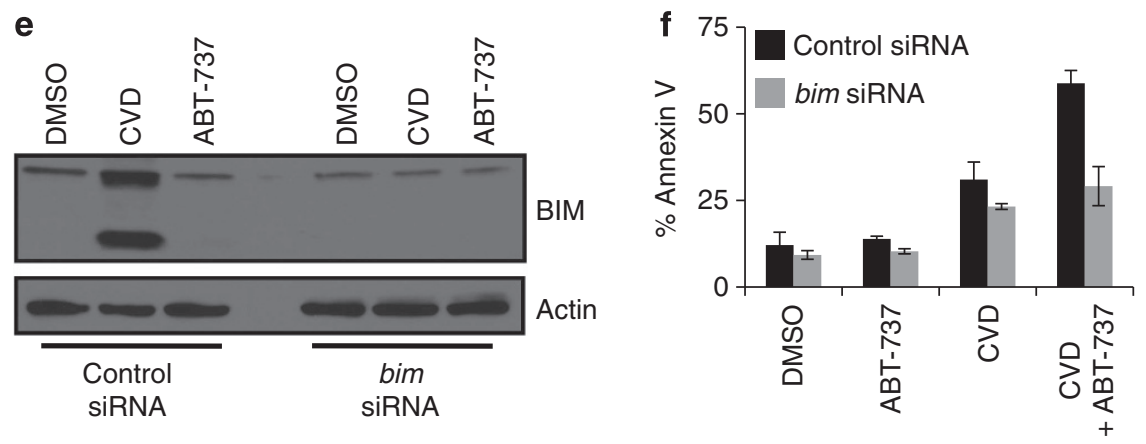

Figure $7 \mathrm{CVD}$ induces BIM expression and function at the OMM, which is revealed by ABT-737. (a) A375 cells were treated with DMSO, ABT-737 (1 $\mu \mathrm{M})$, or the CVD regimen for $48 \mathrm{~h}$, and resulting whole-cell lysates where subjected to SDS-PAGE and western blot for BIM and actin (loading control). (b) A375 cells were treated with ABT-737 $(1 \mu \mathrm{M})$ for $96 \mathrm{~h}$, and the resulting whole-cell lysates were subjected to SDS-PAGE and western blot for BCL-2, BCL-xL, MCL-1, and actin. (c) A375 cells were treated with DMSO or the CVD regimen for $24 \mathrm{~h}$, harvested, permeabilized with $0.015 \%$ digitonin, and treated with C8-BID (10 nM) or ABT-737 $(10,100,1000 \mathrm{~nm})$ for $1 \mathrm{~h}$ at $37^{\circ} \mathrm{C}$ for $1 \mathrm{~h}$. The supernatants were subjected to SDS-PAGE and western blot for cytochrome c. 'Total' cytochrome $\mathrm{c}$ was determined by a detergent treated sample. (d) Untreated A375 cells were permeabilized and processed as in (c). The BIM BH3 domain peptide (10, 100, $1000 \mathrm{~nm}$ ) was added in the presence of DMSO or ABT-737 (1 $\mu$ M). (e) A375 were transfected with siRNAs targeting bim or a control and cultured for $72 \mathrm{~h}$. Whole-cell lysates were subjected to SDS-PAGE and western blot for BIM and actin. (f) The control and bim siRNA lines in (e) were treated with the CVD regimen: dacarbazine $(0.125 \mathrm{~mm})$, vinblastine $(1 \mathrm{~nm})$, and cisplatin $(25 \mu \mathrm{M}) \pm \mathrm{ABT}-737(1 \mu \mathrm{M})$ for $72 \mathrm{~h} \mathrm{before} \mathrm{Annexin} \mathrm{V}$ staining and flow cytometry. All data are reported as \pm S.D. All experiments were performed in triplicate, at least

CVD-induced BIM induction, nor CVD + ABT-737 regulated apoptosis (Figure 7e and f). Taken together, these biochemical and cellular data suggest that BIM expression is necessary and sufficient for the CVD regimen to promote ABT-737-dependent melanoma cell killing.

To examine the chemotherapeutic enhancement of the CVD regimen with ABT-737 in a more physiologically relevant in vivo setting, we established primary A375 melanoma tumors in a chick embryo chorio-allantoic membrane (CAM) assay, and then treated with CVD in the absence or presence of ABT-737. ${ }^{22}$ A375 cells stably expressing mCherry were generated to differentiate the melanoma and chick cells in subsequent assays (Figure 8a). The A375 mCherry cells were then evaluated for their ability to establish a primary tumor in the CAM model. Matrigel containing $2 \times 10^{6}$ A375 mCherry cells was inoculated upon the CAM within a Teflon ring, and the tumor was allowed to develop for 6 days before imaging Matrigel alone resulted in no detectable tumor formation by gross or microscopic analysis of the inoculated region (Figures $8 \mathrm{~b}$ and c). In contrast, Matrigel containing A375 mCherry cells resulted in significant tumor cell engraftment and proliferation within 2-3 days (Figures $8 \mathrm{~b}$ and d). The A375 mCherry tumors were verified by H\&E staining, and were easily distinguishable from the chicken cell environment (Figure $8 \mathrm{~d}$ ). To determine if the CVD regimen plus ABT-737 could influence tumor formation in the CAM model, we established tumors on day 0 , and then treated the tumors as indicated on days 2 and 4, followed by harvesting and analyses on day 6 . DMSO and ABT-737-treated tumors were indistinguishable in both macroscopic and cell number analyses (Figures $8 \mathrm{e}-\mathrm{g}$ ). Tumors treated with CVD were $\sim 25 \%$ smaller than DMSO (Figures $8 \mathrm{e}-\mathrm{g}$ ), which paralleled 


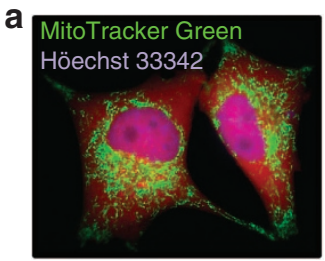

A375 mCherry

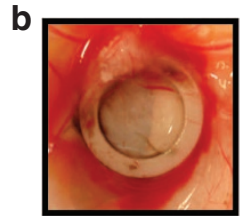

Matrigel (no cells)

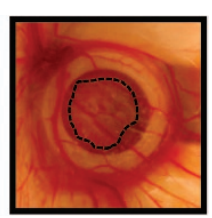

Matrigel + A375 mCherry
C

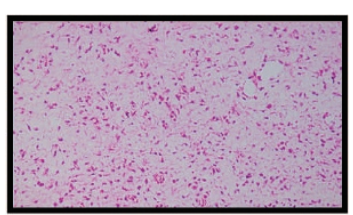

H\&E, Matrigel, 20X

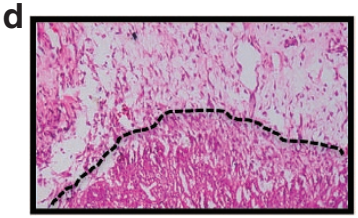

H\&E, A375 mCherry, 20X

e

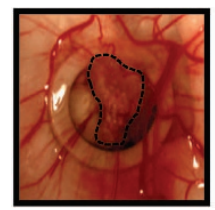

DMSO

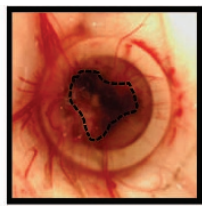

ABT-737

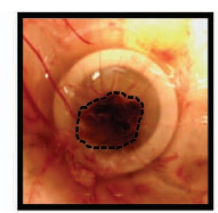

CVD

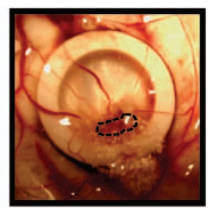

CVD + ABT-737

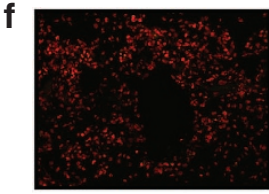

DMSO

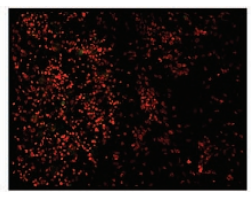

ABT-737

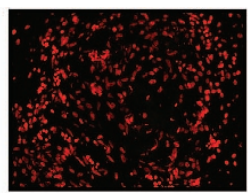

CVD

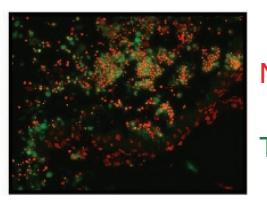

CVD +

ABT-737
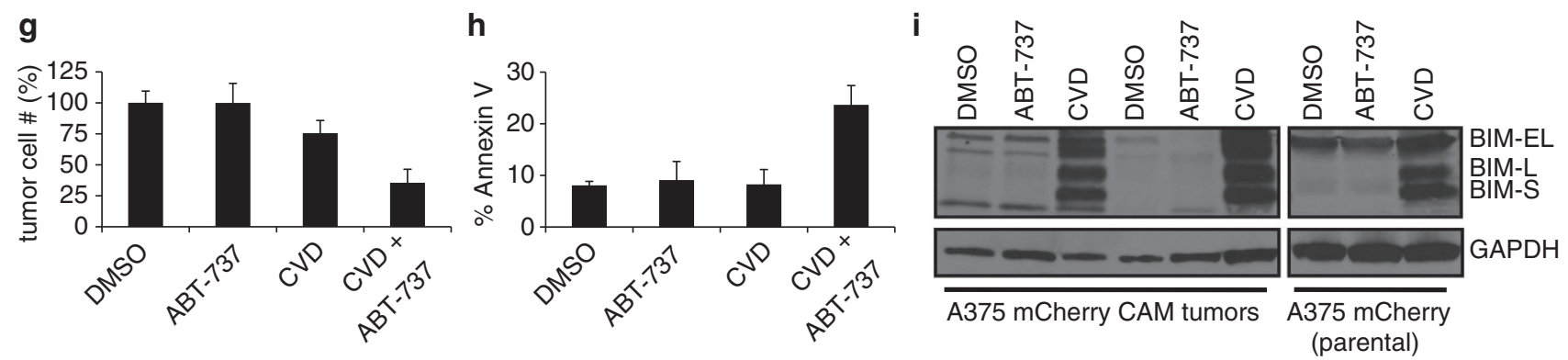

Figure 8 The CVD plus ABT-737 regimen reduces tumor cell number and promotes apoptosis in the CAM model of tumor establishment. (a) The A375 mCherry stable line was co-labeled with MitoTracker Green and Hoechst 33342 to visualize mitochondria and nuclei, respectively, by live cell imaging using a $\times 40$ lens. (b) Matrigel + PBS ${ }^{++}$, or Matrigel $+\mathrm{A} 375 \mathrm{mCherry}$ cells $\left(2 \times 10^{6}\right)$, were inoculated on the CAM and allowed to grow for 6 days prior to gross imaging. Tumors appeared as textured, asymmetric, beige growths within the Teflon rings. The A375 tumor is outlined with black, dashed line. (c, d) Tumors from (b) were excised, fixed, and stained with H\&E. The A375 tumor border is designated with a black, dashed line, although, there is A375 infiltration beyond the border. (e) Representative tumors after indicated treatments. Tumors are outlined with black, dashed lines. N.b., the A375 tumors occasionally become melanotic within the CAM model. (f) Representative tumors after indicated treatments were excised, fixed, and stained for TUNEL. FITC (green) and PI (red) are TUNEL and nuclei, respectively. (g) Tumors from indicated treatments were excised, collagenase treated, and the total number of A375 mCherry cells was determined. (h) Samples in (f) were also stained with Annexin V-FITC, and the \% of Annexin V-FITC positive cells was determined within the mCherry population by flow cytometry. (i) Samples in (f) were pelleted, and resulting whole-cell lysates where subjected to SDS-PAGE and western blot for BIM and GAPDH (a loading control). The tumor samples were compared with the parental A375 cells for BIM induction. All data are reported as \pm S.D. All experiments were performed in triplicate, at least

the inhibition of colony formation observed in Figure 6e. Importantly, ABT-737 markedly sensitized the tumors to the CVD regimen, which resulted in reduced tumor cell number and an appreciable level of apoptosis in vivo, as measured by TUNEL and Annexin V analyses (Figure 8f-h). Previous data suggest that BIM expression was induced by CVD treatment in vitro (Figure $7 \mathrm{a}$ ), and this was required for CVD-induced apoptosis (Figure 7e and f); therefore, we confirmed that the A375 mCherry tumors behaved similarly. As shown in Figure 8i, all CVD-treated tumors demonstrated significant levels of BIM protein, which was not present in DMSO or ABT737-treated samples, and the BIM induction was similar to the parental A375 mCherry line.

\section{Discussion}

As malignant melanoma is highly refractory to conventional chemotherapeutic strategies, we aimed to examine the potential enhancement of tumor cell response rates by collateral inhibition of the anti-apoptotic BCL-2 family members with ABT-737. ${ }^{21}$ The development of ABT-737 as a single agent and adjuvant therapy has been a milestone in cancer treatment as the drug is well tolerated in patients, and has been shown to be effective in numerous tumor types. ${ }^{17,18}$ Patients diagnosed with malignant melanoma are often treated with dacarbazine-based therapies, but the response rates are underwhelming, and for those patients that initially respond, resistance rapidly ensues leaving few clinical options. ${ }^{13-16}$ 
Our data indicate that the dacarbazine-based regimens have minimal effects on melanoma tumor cell proliferation and survival in both murine and human cell lines, which parallels clinical results (Figures 1a, 5a, 6a and b, and $8 f-i) .{ }^{21}$ Despite the lack of an obvious cellular response to dacarbazine or the CVD regimen, co-treatment with $A B T-737$ reveals a proapoptotic program that promotes significant apoptosis (Figures 2, 3, 4, 5b, 6c, and $8 f$ and h). These data suggest that dacarbazine and the CVD regimen are indeed engaging a pro-apoptotic signal, but it remains silenced by anti-apoptotic BCL-2 family repertoire. Moreover, in murine and human cellular models of melanoma, the CVD-induced pro-apoptotic signal is BIM (Figures 5c, 7a and e, and $8 \mathrm{i}$ ), which remains functionally inhibited by anti-apoptotic BCL-2 proteins on the OMM unless ABT-737 is present (Figure 7c and d). We propose that dacarbazine-based therapy response rates and likelihood of resistance may be shifted to favor patient outcome by the introduction of ABT-263, the orally available form of ABT-737, to enhance the pro-apoptotic effect of chemotherapy to eliminate cancer cells from the body and potentially reduce the development of chemoresistance. ${ }^{23}$ Finally, the addition of effective BCL-2 inhibitors to conventional chemotherapeutic regimens may also lower the toxic side effects of treatment as lower doses of chemotherapeutics may provide equivalent tumor responses.

\section{Materials and Methods \\ Reagents. All cell culture and general laboratory reagents were from Invitrogen (Carlsbad, CA, USA) and Sigma (St Louis, MO, USA), respectively. Cisplatin, dacarbazine, and vinblastine were also from Sigma. Annexin V-FITC was produced as described. ${ }^{24}$ Antibodies were from Santa Cruz (Santa Cruz, CA, USA) unless designated (clone): anti-actin (C4), anti-BCL-2 (100), anti-MCL-1 (Rockland, Gilbertsville, PA, USA), anti-BAK (G23), anti-BIM (22-40, Calbiochem, Darmstadt, Germany), anti-BID (C20), anti-cyto c (7H8.2C12), anti-BAK (NT), anti-BAX (N20), anti-BCL-xL (S18), anti-GAPDH (9B3). Caspase-8 cleaved human BID (C8-BID) was from R\&D Systems (Minneapolis, MN, USA). ABT-737 was obtained from Abbott Laboratories (Abbott Park, IL, USA). The BIM BH3 domain peptide (Anaspec, Fremont, CA, USA) was resuspended in DMSO, aliquoted into single use volumes, and stored at $-80^{\circ} \mathrm{C}$. All other recombinant proteins were purified as described. ${ }^{9}$}

Cell culture, apoptosis, clonogenic survival, and proliferation assays. All cells were cultured in DMEM containing 10\% FBS, 2 mM L-glutamine, and antibiotics. For cell death studies, cells were seeded $24 \mathrm{~h}$, treated as described, floating and attached cells were harvested, stained with Annexin V, and analyzed by flow cytometry. For clonogenic survival studies, cells were treated with indicated drugs for $48 \mathrm{~h}$ before changing the media. Colonies were stained with methylene blue 10 days after treatment and imaged. Colonies were then de-stained ( $20 \%$ methanol in $5 \%$ acetic acid), and the supernatant was measured for absorption at $520 \mathrm{~nm}$ for quantification of the wells. STS (100 nM) was often used as a potent inducer of apoptosis. Proliferation was examined using the CyQUANT Direct Cell Proliferation Assay (Invitrogen, measures DNA content). Cells were plated in black wall, clear bottom 96-well plates at a density of $8000 \mathrm{cells} /$ well and allowed to seed for $24 \mathrm{~h}$ before treatment. Following indicated treatments, samples were incubated with the detection reagent for $1 \mathrm{~h}$ at $37^{\circ} \mathrm{C}$ and analyzed for fluorescence (Excitation: $508 \mathrm{~nm}$ and Emission: $527 \mathrm{~nm}$ ). For siRNA studies, cells were transfected with a pool of siRNA oligonucleotides $(10 \mathrm{nmol} / 60 \mathrm{~mm}$ dish, Qiagen (Valencia, CA, USA), FlexiTube for BCL-2L11) targeting bim or mfn2 (control) using Lipofectamine RNAiMAX as described by the manufacturer.

Live cell imaging. Cells were seeded on rat-tail collagen I-coated plates for $24 \mathrm{~h}$ before indicated treatments. Mitochondria and nuclei were labeled with MitoTracker Green (Invitrogen, $100 \mathrm{~nm}$ ) and Hoechst 33342 (Anaspec, $20 \mu \mathrm{m}$ ) for $30 \mathrm{~min}$ at $37^{\circ} \mathrm{C}$. Phenol red free media was used for all imaging performed on a Zeiss Imager.Z1 (Thornwood, NY, USA) equipped with a N-Achroplan $\times 40 / 0.75$ water immersion lens and an AxioCAM MRm digital camera; images were captured using AxioVision 4.8 software (Thornwood, NY, USA).

Western blot analysis. Whole-cell protein lysates were made from trypsinized cells, pelleted, resuspended in RIPA buffer supplemented with protease inhibitors, incubated on ice for $30 \mathrm{~min}$ and centrifuged for $10 \mathrm{~min}$ at $13000 \times g$. Lysates were then adjusted with RIPA buffer to equal the protein concentrations. Proteins were subjected to SDS-PAGE before transferring to nitrocellulose by standard western conditions, blocked in 5\% milk/TBST and primary antibodies (in blocking buffer; actin 1:10000, BAK 1:1000, BAX 1:1000, BCL-2 1:1000, BCL-XL 1:2000, BIM 1:1000, BID 1:500, cytochrome C 1:2000, GAPDH 1:2000) were incubated overnight at $4^{\circ} \mathrm{C}$. The secondary antibody (1:5000 in blocking buffer) was incubated at $25^{\circ} \mathrm{C}$ for $1 \mathrm{~h}$ before standard enhanced chemiluminescence detection.

Cytochrome c release assay. In all, $5 \times 10^{6}$ cells were treated as indicated, trypsinized, and washed twice in cold PBS. The cell pellet was resuspended in permeability buffer ( $250 \mathrm{~mm}$ sucrose, $20 \mathrm{~mm}$ HEPES, pH 7.4, $100 \mathrm{~mm} \mathrm{KCl}, 5 \mathrm{~mm} \mathrm{MgCl}$, $1 \mathrm{~mm}$ EDTA, $1 \mathrm{~mm}$ EGTA, supplemented with $0.015 \%$ digitonin and $1 \times$ HALT Protease and Phosphatase inhibitor cocktail) and incubated on ice for $10 \mathrm{~min}$. The permeabilized pellet was divided into the required number of samples, drugs/proteins were added, and the reactions were incubated at $37^{\circ} \mathrm{C}$ for $1 \mathrm{~h}$. The reactions were then centrifuged at $8000 \times g$ for $10 \mathrm{~min}$, and the supernatants were analyzed for cytochrome $c$ by SDS-PAGE and western blot analysis. Total cytochrome $\mathrm{c}$ was determined by a sample treated with $1 \%$ CHAPS.

CAM assay. Ten-day-old fertilized white Leghorn chicken eggs (Gallus domesticus, SPAFAS) were incubated in a humidified $37^{\circ} \mathrm{C}$ chamber. Under sterile conditions, the eggshell surface was cleaned, a small hole was introduced with a 19-G needle (egg hole punch) in the air sack, and a window was created as described. ${ }^{22,25}$ A375 mCherry cells $\left(2 \times 10^{6}\right.$ cells in $25 \mu$ PBS $^{++}$, or $\mathrm{PBS}^{++}$alone $)$were implanted along with Matrigel $(25 \mu \mathrm{l})$ within a Teflon ring, and allowed to graft for 2 days. $\mathrm{PBS}^{+}+$is $1 \times \mathrm{PBS}$ supplemented with $0.5 \mathrm{~mm} \mathrm{CaCl}$ and $0.5 \mathrm{~mm} \mathrm{MgCl}$. The CAMs were opened on days 2 and 4 for indicated treatments, and harvested on day 6 . Tumor growth was evaluated macroscopically, representative tumors were excised and fixed in 10\% zinc formalin, dehydrated, and embedded in paraffin. Sections $(5 \mu \mathrm{m})$ were processed for hematoxylin and eosin (H\&E) staining or terminal deoxynucleotidyl transferase dUTP nick end labeling (TUNEL). For TUNEL staining, paraffin-embedded tumor specimens were examined for apoptosis using the TUNEL method. The ApopTag Fluorescein In Situ Apoptosis Detection kit (Millipore, Billerica, MA, USA) was used according to the manufacturer's instruction. Prior to the addition of TdT enzyme, sections were deparaffinized with xylene and rehydrated through graded alcohol washes. Antigen retrieval was performed by incubating the sample for $20 \mathrm{~min}$ at room temperature in proteinase K-PBS. Mounting medium with propidium iodide (Vector Laboratories, Burlingame, CA, USA) was used to counterstain, and the tumors were examined and imaged using a Axioplan $2 \mathrm{IE}$ fluorescent microscope (Zeiss, Thornwood, NY, USA). The remaining tumors were type $1 \mathrm{~A}$ collagenase treated, and the trypan blue negative A375 mCherry cells were counted. In parallel, cells were labeled with Annexin V-FITC as indicated above for $\%$ apoptosis. Cells positive for mCherry were gated in this analysis to exclude contaminating chicken cells. Whole-cell lysates from the A375 mCherry tumors were also generated after collagenase treatment as indicated above.

\section{Conflict of Interest}

The authors declare no conflict of interest.

Acknowledgements. We thank everyone in the Chipuk Laboratory for their assistance and support; and Drs. Stuart Aaronson, Emily Bernstein, and Mark Lebwohl for mentorship and guidance in the melanoma field. Dr. Simona Podgrabinska for assistance with microscopy, Dr. Gotham Narla (Case Western Reserve University) for TUNEL assistance, and the Mount Sinai Histology Core. This work was supported by NIH CA157740 (to JEC), the JJR Foundation (to JEC), the William A Spivak Fund (to JEC), the Fridolin Charitable Trust (to JEC), and NIH DE021741 (to AGS).

1. Ibrahim N, Haluska FG. Molecular pathogenesis of cutaneous melanocytic neoplasms. Annu Rev Pathol 2009; 4: 551-579. 
2. Jemal A, Siegel R, Ward E, Hao Y, Xu J, Thun MJ et al. Cancer statistics, 2009. CA Cancer J Clin 2009; 59: 225-249.

3. Letai AG. Diagnosing and exploiting cancer's addiction to blocks in apoptosis. Nat Rev Cancer 2008; 8: 121-132.

4. Chipuk JE, Green DR. How do BCL-2 proteins induce mitochondrial outer membrane permeabilization? Trends Cell Biol 2008; 18: 157-164.

5. Chipuk JE, Moldoveanu T, Llambi F, Parsons MJ, Green DR. The BCL-2 family reunion Mol Cell 2010; 37: 299-310.

6. Kuwana T, Mackey MR, Perkins G, Ellisman MH, Latterich M, Schneiter R et al. Bid, Bax, and lipids cooperate to form supramolecular openings in the outer mitochondrial membrane. Cell 2002; 111: 331-342.

7. Letai A, Bassik MC, Walensky LD, Sorcinelli MD, Weiler S, Korsmeyer SJ et al. Distinct $\mathrm{BH} 3$ domains either sensitize or activate mitochondrial apoptosis, serving as prototype cancer therapeutics. Cancer Cell 2002; 2: 183-192.

8. Kuwana T, Bouchier-Hayes L, Chipuk JE, Bonzon C, Sullivan BA, Green DR et al. BH3 domains of $\mathrm{BH} 3$-only proteins differentially regulate Bax-mediated mitochondrial membrane permeabilization both directly and indirectly. Mol Cell 2005; 17: 525-535.

9. Chipuk JE, Fisher JC, Dillon CP, Kriwacki RW, Kuwana T, Green DR et al. Mechanism of apoptosis induction by inhibition of the anti-apoptotic BCL-2 proteins. Proc Natl Acad SCi USA 2008; 105: 20327-20332.

10. Hocker TL, Singh MK, Tsao H. Melanoma genetics and therapeutic approaches in the 21st century: moving from the benchside to the bedside. $J$ Invest Dermatol 2008; 128 2575-2595.

11. Igney FH, Krammer PH. Death and anti-death: tumour resistance to apoptosis. Nat Rev Cancer 2002; 2: 277-288.

12. Vousden KH, Lane DP. p53 in health and disease. Nat Rev Mol Cell Biol 2007; 8: 275-283.

13. Legha SS, Ring S, Bedikian A, Plager C, Eton O, Buzaid AC et al. Treatment of metastatic melanoma with combined chemotherapy containing cisplatin, vinblastine and dacarbazine (CVD) and biotherapy using interleukin-2 and interferon-alpha. Ann Oncol 1996; 7: 827-835.

14. Legha SS, Ring S, Papadopoulos N, Plager C, Chawla S, Benjamin R et al. A prospective evaluation of a triple-drug regimen containing cisplatin, vinblastine, and dacarbazine (CVD) for metastatic melanoma. Cancer 1989; 64: 2024-2029.
15. Phan GQ, Attia $P$, Steinberg SM, White DE, Rosenberg SA. Factors associated with response to high-dose interleukin-2 in patients with metastatic melanoma. J Clin Oncol 2001; 19: 3477-3482

16. Tsao H, Atkins MB, Sober AJ. Management of cutaneous melanoma. N Engl J Med 2004; 351: $998-1012$.

17. Oltersdorf T, Elmore SW, Shoemaker AR, Armstrong RC, Augeri DJ, Belli BA et al. An inhibitor of Bcl-2 family proteins induces regression of solid tumours. Nature 2005; 435: 677-681.

18. Elkholi R, Floros KV, Chipuk JE. The Role of BH3-Only Proteins in Tumor Cell Development, Signaling, and Treatment. Genes Cancer 2011; 2: 523-537.

19. Fidler IJ. Biological behavior of malignant melanoma cells correlated to their survival in vivo. Cancer Res 1975; 35: 218-224.

20. Overwijk WW, Restifo NP. B16 as a mouse model for human melanoma. Curr Protoc Immunol 2001 Chapter 20: Unit 2021.

21. Anvekar RA, Asciolla JJ, Missert DJ, Chipuk JE. Born to be alive: a role for the BCL-2 family in melanoma tumor cell survival, apoptosis, and treatment. Front Oncol 2011; 34: 1-16.

22. Hagedorn M, Javerzat S, Gilges D, Meyre A, de Lafarge B, Eichmann A et al. Accessing key steps of human tumor progression in vivo by using an avian embryo model. Proc Natl Acad Sci USA 2005; 102: 1643-1648.

23. Tse C, Shoemaker AR, Adickes J, Anderson MG, Chen J, Jin S et al. ABT-263: a potent and orally bioavailable Bcl-2 family inhibitor. Cancer Res 2008; 68: 3421-3428.

24. Logue SE, Elgendy M, Martin SJ. Expression, purification and use of recombinant annexin $\checkmark$ for the detection of apoptotic cells. Nat Protoc 2009; 4: 1383-1395.

25. Ossowski L. In vivo invasion of modified chorioallantoic membrane by tumor cells: the role of cell surface-bound urokinase. J Cell Biol 1988; 107: 2437-2445.

Cell Death and Disease is an open-access journal published by Nature Publishing Group. This work is licensed under the Creative Commons Attribution-NonCommercial-No Derivative Works 3.0 Unported License. To view a copy of this license, visit http://creativecommons.org/licenses/by-nc-nd/3.0/

Supplementary Information accompanies the paper on Cell Death and Disease website (http://www.nature.com/cddis) 